\title{
Increased expression of RNA binding protein GNL3 has potential influence on osteoarthritis by up-regulating IL24 and PTN
}

\author{
Zhen Zhu ${ }^{1}$, Jun Xie ${ }^{1}$, Yawen Bian ${ }^{1}$, Upasana Manandhar ${ }^{1}$, Xiaomin Yao $^{1}$, and Bo Zhang ${ }^{1}$ \\ ${ }^{1}$ Affiliation not available
}

June 3, 2020

\begin{abstract}
Osteoarthritis (OA) is a degenerative joint disorder which affects about $80 \%$ of the population above 65 years revealing radiographic evidence of OA. Recently, more and more researches have been conducted on the molecular mechanism of OA to find target treatments. RNA binding proteins (RBPs) play a key role in genome regulation. Nucleolar GTP-binding Protein 3 (GNL3) is abundantly expressed in bone marrow mesenchymal stem cells and correlates with chondrocytes differentiation. Here we report the transcriptome study of GNL3, which regulates transcription in osteoarthritis (OA). In this study, RNA sequencing (RNA-seq) was used to analyze the global transcription level in HeLa cells. The results showed that the expression of IL24 and PTN was down-regulated when GNL3 was knocked down. Likewise, in the lesions of osteoarthritis, the expression level of GNL3, IL24 and PTN gene were significantly up-regulated compared with the control group. IL24 contributes to the progression of osteoarthritis by inducing bone cells apoptosis at the joint, and PTN contributes to the progression of osteoarthritis by promoting angiogenesis. This study aimed to assess the association between GNL3 and both of these two downstream genes as a potential biomarker for investigating the development of OA.
\end{abstract}

\section{Introduction}

Osteoarthritis (OA) is a degenerative joint disorder characterized by progressive destruction of articular cartilage, synovial hyperplasia and subchondral osteosclerosis [1]. It is one of the main causes of disability in middle-aged and elderly people. With the increase in the aging population and its high medical costs, osteoarthritis and disability caused by it have become an urgent public health issue [2]. The prevalence of diagnosed symptomatic knee osteoarthritis was $4.2 \%^{\sim} 15.5 \%$, which increased with age. About $80 \%$ of the population above 65 years reveals radiographic evidence of KOA [3]. Among the 291 disabilities listed by global burden of disease (GBD), hip and knee OA ranked 11th globally in 2010 and 38th in disability affects life year (DALY) [4]. Although osteoarthritis is considered a disease of the elderly, disabling osteoarthritis can still have a significant impact on work. It is reported that $44.4 \%$ of people who are unemployed due to illness are related to OA in Canada, and the influence is expected to increase over time [5]. In addition to aging, many other factors play a role in the pathogenesis of OA. Both metabolic syndrome and dietary habit play a role in the susceptibility and development of OA [6]. Recently, more and more researches have been conducted on the molecular mechanism of OA to find target treatments for OA. More than 25 miRNAs involved in the occurrence of chondrogenesis and OA have been revealed. In particular, it is regulated by more than one miRNA in chondrocyte formation, chondrocyte differentiation, chondrocyte proliferation, chondrocyte hypertrophy, endochondral ossification and proteolytic enzyme regulation [7]. After knocking down RNA binding-protein zinc finger protein 36 type-like 1 (ZFP36L1) gene, the mRNA expression of two heat shock protein 70 (HSP70) family members increased, the two genes inhibited apoptosis of cartilage, so RNA-binding protein ZFP36L1 plays a role in the pathogenesis of osteoarthritis by modulating members of the HSP70 family, inhibiting chondrocytes apoptosis [8]. However, the specific mechanism of RNA-binding protein in the pathogenesis of osteoarthritis still needs to be clarified. So, the role of RNA-binding protein 
GNL3 was studied in this research in association with the pathogenesis of OA, which can provide new target treatments for OA.

RNA-binding proteins play a key role in post-transcriptional regulation and can affect different stages of RNA metabolism, and process of cell proliferation and apoptosis [9-11]. GNL3 ( Nucleolar GTP-binding Protein 3, Nucleostemin), also known as nucleostem factor, is an RNA-binding protein which plays an important role in cell proliferation, cell cycle regulation, differentiation inhibition, ribosome biosynthesis, stem cell characteristic maintenance, genome stability and telomere integrity [12-14]. It is abundantly expressed in bone marrow mesenchymal stem cells, and correlates with chondrocytes differentiation. GNL3 levels were found to be significantly increased in synovial tissue and fluid of osteoarthritis patients in the clinical experiment [15]. Furthermore, the relationship between GNL3 gene and osteoarthritis in Han Chinese population has also been verified [16]. Further study found that cis-acting regulatory polymorphisms acting on GNL3 contributed to the OA association signal at chromosome 3p21, and the osteoarthritis susceptibility locus had been mapped to 3p21 chromosome [17]. These results proved that GNL3 is closely related to the pathogenesis of osteoarthritis. Therefore, it was speculated that GNL3 is involved in the pathogenesis of osteoarthritis as an RNA-binding protein. So, in this study we explored the role of RNA-binding protein GNL3 in the pathogenesis of osteoarthritis, which can provide a new target for the treatment of osteoarthritis.

In this study, unbiased transcriptome analysis has been utilized to investigate how GNL3 regulates gene transcription in Hela cells. We successfully knocked down the GNL3 gene by shRNA Hela. High-throughput RNA sequencing (RNA-seq) were performed for the experimental group and the control group to obtain the gene expression profiles regulated by GNL3. The results showed evident changes in the transcription profile of specific genes after knocking down the GNL3 gene, some of which were correlated with the incidence of osteoarthritis, including IL24 gene and PTN gene.

IL24 inhibits cell proliferation and angiogenesis, and induces apoptosis in tumor cells [18]. It is determined that IL24 plays an important role in the classical apoptotic pathway p38 [19], and participates in inducing apoptosis in a variety of diseases. It is reported that IL24 reduces the production of dickkopf-1 by fibroblastlike synovial cells in the pathogenesis of spinal arthritis, and also induces osteogenic mineralization, playing a role in aggravating the progression of osteoarthritis [20]. PTN is a secretory growth factor that contributes to the skeletal development of human embryos [21]. Bone epiphysis in the state of ischemia and hypoxia leads to extensive bone cell and tissue death [22], while it is suggested that PTN directly participates in angiogenesis and promotes bone repair [23, 24]. Angiogenesis is essential in the pathogenesis of OA, facilitating the invasion of inflammatory cells and increase in local pain receptors that contribute to structural damage and pain. So, PTN can aggravate the development of osteoarthritis by promoting angiogenesis [25]. In addition, the PTN promotes original bone cell chemotaxis, which can promote the total colony formation of osteoblast, alkaline phosphatase positive colony formation and specificity of alkaline phosphatase activity [26].

Based on previous studies, it was speculated that IL24 mainly aggravates the progression of osteoarthritis by inducing bone cells apoptosis at the joint and PTN may contribute to the progression of osteoarthritis by promoting angiogenesis [19, 24]. While, IL24 and PTN contribute to the progression of osteoarthritis directly, GNL3 promotes the development of osteoarthritis by acting on both of these two downstream genes.

\section{Materials and methods}

ShRNA clones were downloaded from sigma website or designed by Online software.

\subsection{Silence sequence : GTCCTGGTCTTGAACAAGATT}

Sense and antisense strands were annealed to become shRNA. Vector pGFP-B-RS was dissolved by HindIII and BamHI at 37 for $2 \mathrm{~h}^{\sim} 3 \mathrm{~h}$, and enzyme-digested vector was also run on $1.0 \%$ agarose gel and refined by Qiagen column kit. A linearized vector DNA digested by HindIII and BamHI and drop-in shRNA were ligase by T4 DNA Ligase (NEB). Plasmids were brought into Escherichia coli by chemical transformation. Cells were put onto LB plates containing kanamycin. Then plates were incubated overnight at 37 . Colonies were screened by colony PCR (30 cycles) with universal primers (located on the backbone vector). The 
interference sequence of shRNA was checked by Sanger sequencing. CDNA synthesis was done by standard procedures and RT-PCR was performed on the Bio-Rad S1000 with Bestar SYBR Green RT-PCR Master Mix (DBI Bioscience, Shanghai, China). The depth of sequencing information is 10G per specimen.

\subsection{Cell culture and transfections}

Human CC (Cervical Carcinoma) cell lines, HeLa (CCTCC@GDC0009) were obtained from CCTCC (China Center for Type Culture Collection, Wuhan, Hubei, China). HeLa cells were cultured at 37 with $5 \% \mathrm{CO}_{2}$ in Dulbecco's modified Eagle's medium (DMEM) with 10\% fetal bovine serum (FBS), $100 \mu \mathrm{g} / \mathrm{mL}$ streptomycin, and $100 \mathrm{U} / \mathrm{mL}$ penicillin. Plasmid transfection of HeLa cells was operated using Lipofectamine 2000 (Invitrogen, Carlsbad, CA, USA) according to the manufacturer's protocol. Transfected cells were harvested for RT-qPCR analysis after $48 \mathrm{~h}$.

\subsection{MTT assay}

All cells were maintained in low serum, containing 2\% FBS (Invitrogen) with starvation for 10 hours before the experiments were performed. An aliquot of cells $\left(1 *^{*} 10^{3}\right.$ cells/well $)$ was seeded in a 96 -well plate with $2 \%$ FBS and serum-starved for 10 hours, transfected with corresponding vector or siRNA and cultured in normal medium. At 24, 48, 72, and 96 hours after transfection, MTT (3-(4, 5-dimethylthiazol-2-yl)-2, 5diphenyl-tetrazolium bromide) solution $(5 \mathrm{mg} / \mathrm{mL}, 20 \mathrm{~mL})$ was added to each well. After incubation for 4 hours, the medium was removed and $100 \mathrm{~mL}$ dimethyl sulfoxide was added to each well. The relative number of surviving cells was assessed by measuring the optical density of cell lysates at $570 \mathrm{~nm}$. All assays were performed 5 times.

\subsection{Assessment of gene expression}

GAPDH (glyceraldehyde-3-phosphate dehydrogenase) was utilized as a control gene for assessing the effects of GNL3 knockdown. cDNA synthesis was done by standard procedures and RT-qPCR was performed on the Bio-Rad S1000 with Bestar SYBR Green RT-PCR Master Mix (DBI Bioscience, Shanghai, China). The information of primers is presented in Table 1. The compression of each transcript was then standardized to GAPDH mRNA level using 2- $\Delta \Delta \mathrm{CT}$ method [27]. Comparisons were operated with the paired Student's t-test by using GraphPad Prism software (San Diego, CA).

\subsection{RNA extraction and sequencing}

The HeLa cells were triturated into fine powder before RNA extracted with TRIZOL (Ambion). The RNA was further refined with two phenol-chloroform treatments and then preformed with RQ1 DNase (Promega, Madison, WI, USA) to remove DNA. The quality and quantity of the refined RNA were rejudged by measuring the absorbance at $260 \mathrm{~nm} / 280 \mathrm{~nm}$ (A260/A280) using Smartspec Plus (BioRad, USA). The integrity of RNA was further determined by $1.5 \%$ agarose gel electrophoresis.

For each sample, $1 \mu \mathrm{g}$ of the total RNA was utilized for RNA-seq library preparation by VAHTS Stranded mRNA-seq Library Prep Kit (Vazyme). Polyadenylated mRNAs were refined and fragmented, and then translated into double strand cDNA. After the step of end repair and A tailing, the DNAs were roped to VAHTS RNA Adapters (Vazyme). Refined ligation products corresponding to 200-500 bps were digested with heat-labile UDG, and before sequencing, the single strand cDNA was amplified, refined, quantified and stored at -80 .

The libraries were prepared following the manufacturer's instructions and applied to Illumina HiSeq X Ten system for $150 \mathrm{nt}$ paired-end sequencing for high-throughput sequencing.

\subsection{RNA-Seq Raw Data Clean and Alignment}

Raw reads covering more than $2-\mathrm{N}$ bases were first cast off. Then adaptors and low-quality bases were removed from raw sequencing reads using FASTX-Toolkit (Version 0.0.13). The short reads less than 16nt were also discarded. Then, clean reads were aligned to the GRch38 genome by tophat2 [28] allowing 4 
mismatches. Uniquely mapped reads were utilized for gene reads number counting and FPKM calculation (fragments per kilobase of transcript per million fragments mapped) [29].

\subsection{Differentially Expressed Genes (DEG) analysis}

The R Bioconductor package edgeR [30] was used to screen out the differentially expressed genes (DEGs). A false discovery rate $<0.05$ and fold change $>2$ or $<0.5$ were set as the cut-off criteria for identifying DEGs.

\subsection{Reverse transcription q-PCR validation of DEGs}

In this work, to elucidate the validity of the RNA-seq data, quantitative reverse-transcription polymerase chain reaction (q-RT-PCR) was performed for some of the DEGs. The information of primers is presented in Table 4. Total RNA remaining from RNA-seq library preparation was utilized for q-RT-PCR. RNA was reverse transcribed into cDNA utilizing M-MLV Reverse Transcriptase (Vazyme). Real-time PCR was performed with the StepOne RealTime PCR System using the SYBR Green PCR Reagents Kit (Yeasen). The PCR conditions compose of denaturing at $95 @ \mathrm{C}$ for $10 \mathrm{~min}, 40$ cycles of denaturing at $95 @ \mathrm{C}$ for 15 $\mathrm{s}$, annealing and extension at $60 @ \mathrm{C}$ for $1 \mathrm{~min}$. For each sample, PCR amplifications were performed in triplicate. The RNA expression levels of all the genes were standardized against that of GAPDH.

\subsection{Functional enrichment analysis}

To pick out functional categories of DEGs, Gene Ontology (GO) terms and KEGG pathways were identified using KOBAS 2.0 server [31]. Hypergeometric test and Benjamini-Hochberg FDR controlling procedure were utilized to define the enrichment of each term.

\subsection{Clinical verification}

Femoral head samples were collected from 20 patients with OA and set-matched biopsies in 20 patients who had joint replacement surgery caused by femoral neck fracture at Wuhan Union Hospital, Huazhong University of Science and Technology. This study was approved by the Medical Ethics Committee of Huazhong University of Science and Technology Tongji Medicine College under protocol number (2019) IEC (S531). Written informed consent was obtained from each participant prior to sample collection, and all experiments were performed in accordance with relevant guidelines and regulations. The following inclusion criteria were used: Osteoarthritis was diagnosed according to the American rheumatology association's diagnostic criteria and WOMAC scale. And the control group was selected patients with joint replacement surgery caused by femoral neck fracture excluded joint lesions caused by gout, rheumatoid arthritis, steroid-induced necrosis and alcohol-induced necrosis. The informed consent form was in the Supporting Information.

\section{Results}

\subsection{GNL3 promotes cell proliferation in HeLa cells}

In order to verify the effect of knockdown GNL3 on cell reproduction and apoptosis, cytological experiments were carried out. After 48 hours of transfection, the green fluorescent protein (GFP) fluorescence expression after shRNA transfection revealed that the cell transfection was successful (Figure 1A). The reproduction and apoptosis of cells in the experimental and the control groups were detected by MTT assay and apoptosis kit, respectively. The results showed that cell reproduction was inhibited while cell apoptosis was enhanced in the GNL3-knockdown group when compared with the control group (Figure 1B and C).

\section{RNA-seq analysis of GNL3-regulated genes in HeLa cell}

In this experiment, cDNA libraries and high-throughput sequencing of samples have been successfully constructed. The library was constructed using Illunima HiSeq X Ten sequencing platform and paired with end sequencing, and high quality transcriptome data were obtained. In this study, after knocking down GNL3, the GNL3 expression in the experimental group decreased significantly (Figure 2A and B), which indicated that the modeling was successful. 
To ensure the reliability of the experimental data, quality control on the sequencing results were conducted (Table 2). As shown in table 2, raw reads per sample were generated and clean reads per sample were retained by the removal of adaptors and contaminating sequences. Among these, the data of Clean Per in these four groups were all more than $95 \%$, which means that the polluted possibility of this sequencing was little.

The samples in this experiment were from HeLa cell line, Homo sapiens, and the reference genome version was GRCh38. We selected software TopHat 2 [28]. After a certain tolerance rate was set, clean reads was matched to the reference genome (Table 3). Total mapped indicates the number and proportion of reads that can be located in the genome. In transcriptome sequencing, if there is no contamination and the reference genome is selected appropriately, the proportion of this part of data is usually higher than $70 \%$. The data in the four groups of this experiment were all above $90 \%$ which indicated that the data in this experiment was reliable. As for the data analysis on the downstream, only this batch of reads was used to ensure the reliability of the experimental results.

In order to compare the differences in gene expression among different samples, FPKM value was used to represent gene expression. FPKM (expected fragments per kilobase of transcript per million fragments mapped) represents the number of reads per million base lengths from a gene. In this research, most genes were at an expression level of FPKM [?] 20 (Figure 2D). Then the differential expression of transcriptome between samples were analyzed (DEG). First, inter-sample clustering analysis on the correlation between samples was conducted (Figure 2C) to determine the correctness of sampling.

Based on the detections above, samples were compared and differentially expressed genes (DEG) were obtained. Software edgeR was selected to analyze the differential expression between two or more samples. The fold change (FC) and false discovery rate (FDR) were used to determine whether a gene was differentially expressed. The evaluation criteria for significant difference expression were FC [?] 2 or [?] 0.5,FDR $<0.05$. Through the comparison between the experimental group and the control group, it is indicated that GNL3 extensively regulated gene transcription. Heatmap analysis of the expression patterns of partial DEGs in RNA-seq samples showed a high consistency of the GNL3-mediated transcription in both data sets (Figure $2 \mathrm{E})$.

\section{Functional clustering of GNL3-regulated genes}

In order to reveal the biological role of these DEGs, a taxonomic enrichment analysis of the differentially expressed genes on GO and KEGG was performed (Figure 3A and B). In the Figure 3, the top ten representative events enriched in the biological processes subset of GO and pathways of KEGG were selected. As shown in Figure 3 after knockdown of GNL3, the down-regulated gene functions are concentrated in the aspects of signal transduction, apoptotic, transcription, cell proliferation and primary immunodeficiency. Through enrichment analysis on GO and KEGG, it was speculated that GNL3 plays an important role in a series of biological activities such as inflammation and angiogenesis.

Interestingly, it was found that GNL3 knockdown significantly affected the expression of many genes including IL24 gene and PTN gene (Figure 3C and D). Q-RT-PCR was performed to verify the expression of IL24 and PTN (Figure 3E and F). The results showed that the expression of IL24 and PTN was down-regulated when GNL3 was knocked down.

\section{Expression level of GNL3, IL24 and PTN gene in the OA patients}

To further verify the results, femoral head specimens from 20 patients with osteoarthritis and 20 control samples were collected (Figure 4A and B). The results showed that the expression level of GNL3, IL24 and PTN gene in specimens were significantly up-regulated compared with the control group (Figure 4C and D). GNL3 is abundantly expressed in bone marrow mesenchymal stem cells and correlates with chondrocytes differentiation. IL24 induces apoptosis and PTN promotes angiogenesis respectively in the pathogenesis of osteoarthritis. So, the expression of IL24 and PTN genes were up-regulated with OA.

\section{Discussion}


Through previous studies, it is known that osteoarthritis is a degenerative bone and joint disorder. Although aging and obesity are closely related to the onset of $\mathrm{OA}[32,33]$, the specific mechanism has not yet been clarified. This study investigated the role of RNA-binding protein GNL3 in the pathogenesis of osteoarthritis. It is found that GNL3 indirectly affected the pathogenesis of osteoarthritis by affecting the transcription of downstream genes. So this study has provided new therapeutic targets of osteoarthritis.

GNL3 (Nucleolar GTP-binding Protein 3), also known as nucleostemin, plays an important role in cell proliferation, cell cycle regulation and other aspects $[12,13]$. Although previous studies showed that the expression of GNL3 gene increased in OA rats and Chinese Han population [16], the specific molecular mechanism has not been clarified. We considered multiple possibilities of the potential mechanism of GNL3 in OA. There have been few previous studies on GNL3 as an RNA-binding protein, and some studies have confirmed that GNL3 interacts with the susceptibility gene loci of osteoarthritis [34]. Therefore, we speculated that RNA-binding protein GNL3 affects the transcription and expression of downstream genes thus affecting the incidence of osteoarthritis.

In this experiment, high-throughput RNA sequencing (RNA-seq) was performed on the experimental and control groups after GNL3 gene was knocked down in Hela cells to obtain complete transcriptome information. Unlike traditional hybridization methods, RNA-seq has several advantages. First of all, RNA-seq is not limited to detecting transcripts corresponding to existing genome sequences. For example, 454-based RNAseq was used to sequence the transcriptome of Glanville fritillary butterfly [35]. RNA-seq also revealed changes in the sequence of transcriptional regions [36, 37]. RNA-seq has no quantification upper limit, which is associated with the number of sequences obtained. So it can detect expression levels over a large dynamic range. A greater than 9,000-fold range was measured in a study that analyzed 16 million mapped reads in Saccharomyces cerevisiae [38], and a range spanning five orders of magnitude was measured for 40 million mouse sequence reads [39]. RNA-seq is controlled at very precise quantifying expression levels using quantitative PCR (q-PCR) and spike-in RNA of known concentrations [38] making the results highly reproducible for both technical and biological replicates [36, 38].

It was observed that during GNL3 gene knock-down, a large number of genes showed differential expression, indicating that GNL3 extensively regulated gene expression. The functions of these genes were clustered into GO and KEGG databases, and interestingly the results showed that the functions of down-regulated genes were mainly in signal transduction, apoptotic, transcription, cell proliferation and primary immunodeficiency. The DEGs were comprised in pathways such as Jak-STAT signality pathway, p53 pathway, p53-Akt pathway, transcription misregulation in cancer, NF-kappa B signality pathway and cell adhesion molecules. Notably the two down-regulated genes, IL24 gene and PTN gene, have been closely associated with the development of osteoarthritis in previous researches. It is obviously that PTN is upregulated in our eight sets of experiments. To draw the heat-map, we only selected two appropriate sets of results where PTN gene up-regulation was not significant.

In this work, it is confirmed that RNA-binding protein GNL3 affected the expression of downstream genes, including IL24 and PTN, by affecting RNA transcription. IL24 and PTN play an important role in promoting the development of osteoarthritis. To further prove the role of GNL3 in the pathogenesis of osteoarthritis, the local tissues of OA patients and the control groups were selected for q-RT-PCR. The results are shown as the mean \pm standard deviation of three genes. It indicates that there is a significant difference in gene expression between the OA groups and the control groups. The expression level of IL24 gene in OA patients was nearly twice higher than control group insisting that it mainly aggravates the inflammatory response at the joint site in osteoarthritis. IL24 is an immune-regulating cytokine with a wide range of tumor-specific inhibitory effects [40,41], and it induces apoptosis through intracellular and extracellular signaling pathways [42]. It has also been observed that IL24 induces metaphase chromosome protein 1 (MCP1) production in synovial mononuclear cells, which aggravates the inflammatory response and promotes the development of spinal joint arthritis and rheumatoid arthritis [43]. Similarly, as PTN is responsible for promoting angiogenesis, the expression level of PTN gene in OA patients was nearly seen to be seven times higher than that in control group. PTN is a secretory polypeptide that plays an important role in cell growth, migration and 
angiogenesis $[44,45]$. In addition to directly promoting angiogenesis, PTN also influences the behavior of other cells by promoting the proliferation and migration of endothelial progenitor cells [46], and promotes the formation of angiogenic microenvironment [47]. Angiogenesis is one of the earliest histopathological changes in chronic non-communicable arthritis [48]. GNL3 was also expressed about seven times higher than that in control group. Hence, from the results observed in HeLa cells and in the specimens of OA patients, GNL3 developed osteoarthritis by acting on both of these two downstream genes.

GNL3 affects the JAK-STAT pathway through its interaction with Signal Transducer And Activator Of Transcription 3 (STAT3) [49]. It has been shown that when STAT3 was activated, the expression level of downstream gene IL24 increased [50]. Binding anaplastic lymphoma kinase (ALK) which induces MAPK pathway activation is an important step in the anti- apoptotic signaling of PTN and regulation of cell proliferation [51]. STAT3 inhibitor can inhibit the function of ALK, thereby reducing the expression of PTN gene [52]. Therefore, we believe that after knocking down GNL3, expression levels of IL24 and PTN genes in the JAK-STAT pathway are reduced through the interaction with STAT3 gene.

\section{Acknowledgement}

We gratefully acknowledge funding for this work provided by National Natural Science Foundation of China (81501888 and 81802722). This study was partially supported by ABLife (ABL-7702069).

\section{Conflict of interest}

The authors declare no financial or commercial conflict of interest.

\section{References}

[1] B. Mandelbaum, D. Waddell, Orthopedics 2005 ,28, s207-14.

[2] R. C. Lawrence, D. T. Felson, C. G. Helmick, L. M. Arnold, H. Choi, R. A. Deyo, S. Gabriel, R. Hirsch, M. C. Hochberg, G. G. Hunder, J. M. Jordan, J. N. Katz, H. M. Kremers, F. Wolfe, W. National Arthritis Data, Arthritis Rheum 2008 , 58 , 26-35.

[3] D. Huang, Y. Q. Liu, L. S. Liang, X. W. Lin, T. Song, Z. G. Zhuang, S. L. Wang, H. G. Bao, L. Wang, X. W. Zhang, Z. G. Cheng, B. L. Duan, W. D. Qiu, Y. C. Xiong, J. F. Liu, Pain Res Manag2018, 2018 , 2010129.

[4] M. Cross, E. Smith, D. Hoy, S. Nolte, I. Ackerman, M. Fransen, L. Bridgett, S. Williams, F. Guillemin, C. L. Hill, L. L. Laslett, G. Jones, F. Cicuttini, R. Osborne, T. Vos, R. Buchbinder, A. Woolf, L. March, Ann Rheum Dis 2014, 73 , 1323-1330.

[5] B. Sharif, R. Garner, D. Hennessy, C. Sanmartin, W. M. Flanagan, D. A. Marshall, Osteoarthritis Cartilage 2017 ,25, 249-258.

[6] A. Bortoluzzi, F. Furini, C. A. Scire, Autoimmun Rev2018 , 17, 1097-1104.

[7] C. Wu, B. Tian, X. Qu, F. Liu, T. Tang, A. Qin, Z. Zhu, K. Dai,Int J Mol Med 2014, 34, 13-23.

[8] Y. O. Son, H. E. Kim, W. S. Choi, C. H. Chun, J. S. Chun,Nat Commun 2019, 10 , 77.

[9] C. H. de Moor, J. D. Richter, Int Rev Cytol2001, 203 , 567-608.

[10] Q. Wang, F. Wang, W. Zhong, H. Ling, J. Wang, J. Cui, T. Xie, S. Wen, J. Chen, Gene 2019 , 697 , $26-34$.

[11] R. Z. Li, J. Hou, Y. Wei, X. Luo, Y. Ye, Y. Zhang, Gene2019 , 687 , 125-134.

[12] L. Meng, T. Lin, G. Peng, J. K. Hsu, S. Lee, S. Y. Lin, R. Y. Tsai, Proc Natl Acad Sci U S A 2013 , $110,11415-20$.

[13] Q. Zhu, H. Yasumoto, R. Y. Tsai, Mol Cell Biol2006 , 26 , 9279-90. 
[14] A. Castello, B. Fischer, K. Eichelbaum, R. Horos, B. M. Beckmann, C. Strein, N. E. Davey, D. T. Humphreys, T. Preiss, L. M. Steinmetz, J. Krijgsveld, M. W. Hentze, Cell 2012 ,149, 1393-406.

[15] M. L. Louka, Z. M. Zakaria, M. M. Nagaty, M. A. Elsebaie, L. M. Nabil, Gene 2016 , 587, 27-32.

[16] B. Liu, H. Cheng, W. Ma, F. Gong, X. Wang, N. Duan, X. Dang,Sci Rep 2018 , 8, 9610

[17] F. Gee, C. F. Clubbs, E. V. Raine, L. N. Reynard, J. Loughlin,BMC Med Genet 2014 , 15 , 53.

[18] B. Liu, F. Chen, Y. Wu, X. Wang, M. Feng, Z. Li, M. Zhou, Y. Wang, L. Wu, X. Liu, D. Liang, Oncotarget $2017,8,40791-40803$.

[19] M. Ma, L. Zhao, G. Sun, C. Zhang, L. Liu, Y. Du, X. Yang, B. Shan, Oncol Rep 2016 , 35 , 3122-3130.

[20] T. W. Kragstrup, M. N. Andersen, B. Schiottz-Christensen, A. G. Jurik, M. Hvid, B. Deleuran, Clin Exp Immunol $2017,189,342-351$.

[21] M. Lamprou, A. Kaspiris, E. Panagiotopoulos, P. V. Giannoudis, E. Papadimitriou, Injury 2014 , 45 , 1816-1823.

[22] H. K. Kim, H. Bian, J. Aya-ay, A. Garces, E. F. Morgan, S. R. Gilbert, Bone 2009 , 45 , 280-288.

[23] A. Kaspiris, C. Mikelis, M. Heroult, L. Khaldi, T. B. Grivas, I. Kouvaras, S. Dangas, E. Vasiliadis, F. Liote, J. Courty, E. Papadimitriou, Joint Bone Spine 2013 , 80 , 407-413.

[24] A. Kaspiris, E. Chronopoulos, T. B. Grivas, E. Vasiliadis, L. Khaldi, M. Lamprou, P. P. Lelovas, N. Papaioannou, I. A. Dontas, E. Papadimitriou, Cytokine 2016 , 78 , 7-15

[25] I. J. MacDonald, S. C. Liu, C. M. Su, Y. H. Wang, C. H. Tsai, C. H. Tang, Int J Mol Sci ,DOI:10.3390/ijms19072012.

[26] X. Yang, R. S. Tare, K. A. Partridge, H. I. Roach, N. M. Clarke, S. M. Howdle, K. M. Shakesheff, R. O. Oreffo, J Bone Miner Res 2003, 18, 47-57.

[27] K. J. Livak, T. D. Schmittgen, Methods 2001 ,25 , 402-408.

[28] D. Kim, G. Pertea, C. Trapnell, H. Pimentel, R. Kelley, S. L. Salzberg, Genome Biol 2013 , 14 , R36.

[29] C. Trapnell, B. A. Williams, G. Pertea, A. Mortazavi, G. Kwan, M. J. van Baren, S. L. Salzberg, B. J. Wold, L. Pachter, Nat Biotechnol 2010 , 28 , 511-515.

[30] M. D. Robinson, D. J. McCarthy, G. K. Smyth,Bioinformatics 2010 , 26 , 139-140.

[31] C. Xie, X. Mao, J. Huang, Y. Ding, J. Wu, S. Dong, L. Kong, G. Gao, C. Y. Li, L. Wei, Nucleic Acids Res 2011 , 39, W316-322.

[32] L. A. Mandl, Osteoarthritis Cartilage 2019 ,27, 359-364.

[33] T. W. O’Neill, P. S. McCabe, J. McBeth, Best Pract Res Clin Rheumatol 2018 , 32 , 312-326.

[34] O. C. arc, O. C. arc, E. Zeggini, K. Panoutsopoulou, L. Southam, N. W. Rayner, A. G. Day-Williams, M. C. Lopes, V. Boraska, T. Esko, E. Evangelou, A. Hoffman, J. J. Houwing-Duistermaat, T. Ingvarsson, I. Jonsdottir, H. Jonnson, H. J. Kerkhof, M. Kloppenburg, S. D. Bos, M. Mangino, S. Metrustry, P. E. Slagboom, G. Thorleifsson, E. V. Raine, M. Ratnayake, M. Ricketts, C. Beazley, H. Blackburn, S. Bumpstead, K. S. Elliott, S. E. Hunt, S. C. Potter, S. Y. Shin, V. K. Yadav, G. Zhai, K. Sherburn, K. Dixon, E. Arden, N. Aslam, P. K. Battley, I. Carluke, S. Doherty, A. Gordon, J. Joseph, R. Keen, N. C. Koller, S. Mitchell, F. O'Neill, E. Paling, M. R. Reed, F. Rivadeneira, D. Swift, K. Walker, B. Watkins, M. Wheeler, F. Birrell, J. P. Ioannidis, I. Meulenbelt, A. Metspalu, A. Rai, D. Salter, K. Stefansson, U. Stykarsdottir, A. G. Uitterlinden, J. B. van Meurs, K. Chapman, P. Deloukas, W. E. Ollier, G. A. Wallis, N. Arden, A. Carr, M. Doherty, A. McCaskie, J. M. Willkinson, S. H. Ralston, A. M. Valdes, T. D. Spector, J. Loughlin, Lancet 2012, 380 , 815-823. 
[35] J. C. Vera, C. W. Wheat, H. W. Fescemyer, M. J. Frilander, D. L. Crawford, I. Hanski, J. H. Marden, Mol Ecol 2008 ,17, 1636-1647.

[36] N. Cloonan, A. R. Forrest, G. Kolle, B. B. Gardiner, G. J. Faulkner, M. K. Brown, D. F. Taylor, A. L. Steptoe, S. Wani, G. Bethel, A. J. Robertson, A. C. Perkins, S. J. Bruce, C. C. Lee, S. S. Ranade, H. E. Peckham, J. M. Manning, K. J. McKernan, S. M. Grimmond, Nat Methods 2008 , 5 , 613-619.

[37] R. Morin, M. Bainbridge, A. Fejes, M. Hirst, M. Krzywinski, T. Pugh, H. McDonald, R. Varhol, S. Jones, M. Marra, Biotechniques 2008 , 45 , 81-94.

[38] U. Nagalakshmi, Z. Wang, K. Waern, C. Shou, D. Raha, M. Gerstein, M. Snyder, Science 2008 , 320 , 1344-1349.

[39] A. Mortazavi, B. A. Williams, K. McCue, L. Schaeffer, B. Wold,Nat Methods 2008 , 5 , 621-628.

[40] C. C. Cunningham, S. Chada, J. A. Merritt, A. Tong, N. Senzer, Y. Zhang, A. Mhashilkar, K. Parker, S. Vukelja, D. Richards, J. Hood, K. Coffee, J. Nemunaitis, Mol Ther 2005 , 11 , 149-159.

[41] A. W. Tong, J. Nemunaitis, D. Su, Y. Zhang, C. Cunningham, N. Senzer, G. Netto, D. Rich, A. Mhashilkar, K. Parker, K. Coffee, R. Ramesh, S. Ekmekcioglu, E. A. Grimm, J. van Wart Hood, J. Merritt, S. Chada, Mol Ther 2005 , 11, 160-172.

[42] M. Sauane, I. V. Lebedeva, Z. Z. Su, H. T. Choo, A. Randolph, K. Valerie, P. Dent, R. V. Gopalkrishnan, P. B. Fisher, Cancer Res 2004, 64, 2988-2993.

[43] T. W. Kragstrup, K. Otkjaer, C. Holm, A. Jorgensen, M. Hokland, L. Iversen, B. Deleuran, Cytokine 2008, $41,16-23$

[44] C. Mikelis, M. Koutsioumpa, E. Papadimitriou, Recent Pat Anticancer Drug Discov 2007 , 2 , 175-186.

[45] E. Papadimitriou, C. Mikelis, E. Lampropoulou, M. Koutsioumpa, K. Theochari, S. Tsirmoula, C. Theodoropoulou, M. Lamprou, E. Sfaelou, D. Vourtsis, P. Boudouris, Eur Cytokine Netw 2009 ,20 , 180190.

[46] C. Heiss, M. L. Wong, V. I. Block, D. Lao, W. M. Real, Y. Yeghiazarians, R. J. Lee, M. L. Springer, J Cell Physiol2008, 215, 366-373.

[47] B. G. Sharifi, Z. Zeng, L. Wang, L. Song, H. Chen, M. Qin, M. R. Sierra-Honigmann, S. WachsmannHogiu, P. K. Shah, Arterioscler Thromb Vasc Biol 2006 , 26 , 1273-1280.

[48] I. B. McInnes, G. Schett, N Engl J Med 2011,365 , 2205-2219.

[49] J. Wang, K. Huo, L. Ma, L. Tang, D. Li, X. Huang, Y. Yuan, C. Li, W. Wang, W. Guan, H. Chen, C. Jin, J. Wei, W. Zhang, Y. Yang, Q. Liu, Y. Zhou, C. Zhang, Z. Wu, W. Xu, Y. Zhang, T. Liu, D. Yu, Y. Zhang, L. Chen, D. Zhu, X. Zhong, L. Kang, X. Gan, X. Yu, Q. Ma, J. Yan, L. Zhou, Z. Liu, Y. Zhu, T. Zhou, F. He, X. Yang, Mol Syst Biol2017 , 13, 965.

[50] J. Y. Kim, J. C. Kim, J. Y. Lee, M. J. Park, Int J Oncol2018, 53 , 47-58.

[51] P. Perez-Pinera, W. Zhang, Y. Chang, J. A. Vega, T. F. Deuel,J Biol Chem 2007, 282 , 28683-28690.

[52] K. Takezawa, I. Okamoto, K. Nishio, P. A. Janne, K. Nakagawa, Clin Cancer Res 2011 , 17 , 2140-2148.

Table 1. q-RT-PCR primers for gene expression qualification.

\begin{tabular}{lll}
\hline Gene & Primer & Sequence (5'-3') \\
\hline GAPDH & Forward & CGGAGTCAACGGATTTGGTCGTAT \\
& Reverse & AGCCTTCTCCATGGTGGTGAAGAC \\
GNL3 & $\begin{array}{l}\text { Forward } \\
\text { Reverse }\end{array}$ & TGAGGTGTTGGATGCCAGAG \\
& TGACACCACTGTTGGCAAT
\end{tabular}




\begin{tabular}{lll}
\hline Gene & Primer & Sequence (5'-3') \\
\hline PTN & Forward & GAGAATGGCAGTGGAGTG \\
& Reverse & GCTTGGAGATGGTGACAG \\
IL24 & $\begin{array}{l}\text { Forward } \\
\text { Reverse }\end{array}$ & TCTCATCGTGTCACAACTG \\
& ATCAGTCAGAAGAATGTC \\
\hline
\end{tabular}

Table 2. High quality effective sequences

\begin{tabular}{lllll}
\hline SampleID & Raw & Clean & Clean Per & Unique Tag \\
\hline shGnl3 & 76707134 & 73102684 & $95.30 \%$ & $28316498(38.74 \%)$ \\
shCtrl & 78078976 & 74920851 & $95.96 \%$ & $30932962(41.29 \%)$ \\
\hline
\end{tabular}

a) Sample ID: Sample number. b) Raw: Raw reads, the number of original sequences from the original image data obtained by sequencing and converted by base calling. c) Clean: Clean reads, raw reads were removed from the adaptor sequence and low-quality bases, and the number of effective sequences was used for subsequent analysis. d) Clean Per: the proportion of Clean reads in raw reads. e) Unique tag: Unique tag, number of non-repeat reads and proportion of clean reads.

Table 3. Effective sequencing data were compared to the reference genome

\begin{tabular}{lll}
\hline Sample & shCtrl & shGnl3 \\
\hline Total reads & 72284154 & 70622804 \\
Total mapped & $66382194(91.84 \%)$ & $66780627(94.56 \%)$ \\
Total Uniquely mapped & $64232872(96.76 \%)$ & $64926867(97.22 \%)$ \\
Total Multiple mapped & $2149322(3.24 \%)$ & $1853760(2.78 \%)$ \\
\hline
\end{tabular}

a) Total reads: the number of pairs of reads in clean reads, which are used for subsequent analysis.b) Total mapped: the number and proportion of reads that have been mapped to the genome. c) Total mapped reads: the number of reads uniquely located in the reference genome and the proportion of reads in Total mapped reads. d) Total multiple reads: the number of reads with Multiple locations in the reference genome and the proportion of reads in Total mapped reads. Reads with Multiple locations are mainly rRNA and tRNA.

\section{Figure legends}

Figure 1. GNL3 promotes cell viability. (A) GFP fluorescence expression after transfection for 48 hours. (B)MTT assay revealed that GNL3 knockdown inhibited cell reproduction of HeLa cells. P-value in Rep1 and Rep2 sets are respectively 0.000268 and $0.0107 .{ }^{*} \mathrm{P}<0.10,{ }^{* * *} \mathrm{P}<0.01$ compared to the control groups.

(C) Flow cytometer revealed that GNL3 knockdown promoted cell apoptosis of HeLa cells. 


\section{A}

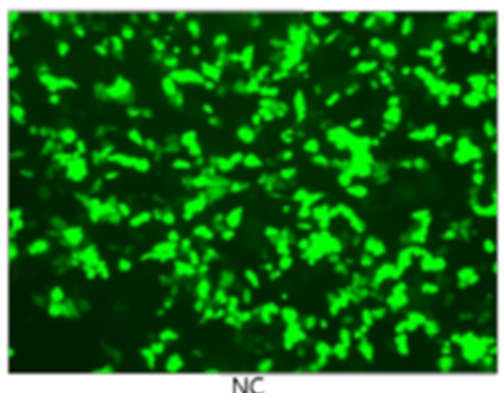

B

Growth of Hela cells

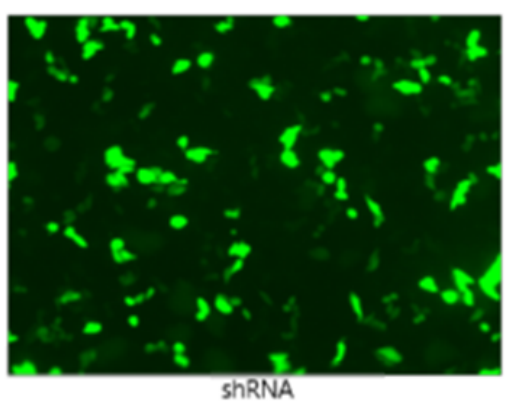

C Apoptosis of Hela cells
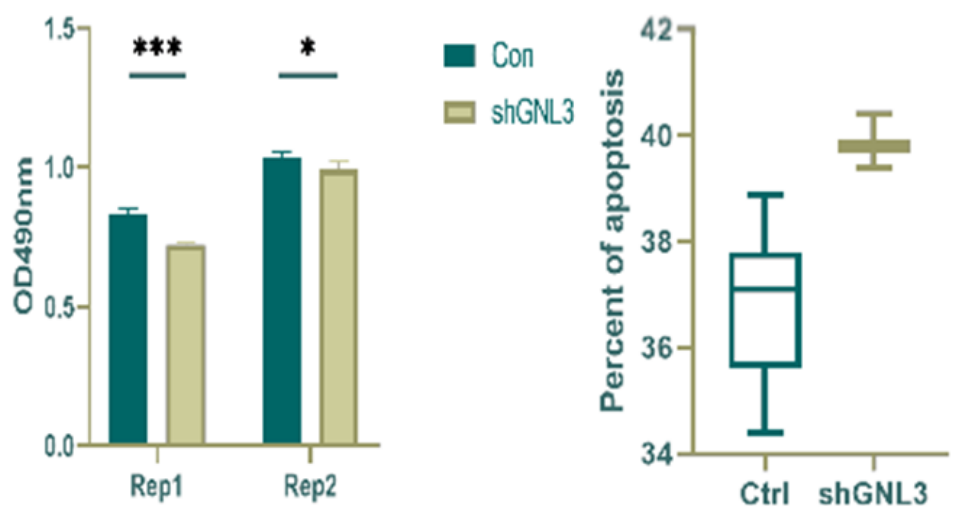

Figure 2. RNA-seq analysis of GNL3-regulated genes in HeLa cells. (A) GNL3 expression quantified by RNA sequencing data. FPKM values were calculated as that has been explained in Materials and Methods. P-value is 0.00441. (B) The result of GNL3 q-RT-PCR analysis. P-value is 4.604E-10. (C) Identification of GNL3 regulated genes. Up-regulated genes are labeled in red, whereas down-regulated are labeled in blue in the heat map. (D) Cumulative distribution of FPKM values of all detected genes in the sample. The horizontal coordinate represents the FPKM value, the vertical coordinate on the left represents the number of genes, and the vertical coordinate on the right represents the proportion of genes. For each point on the curve, its vertical coordinate represents the number and proportion of genes whose FPKM value is less than or equal to the horizontal coordinate of this point. (E) Heat map shows the hierarchically clustered Pearson correlation matrix resulted from comparing the transcript expression values for control and GNL3 knockdown samples. 
A

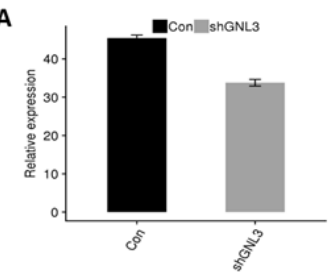

D
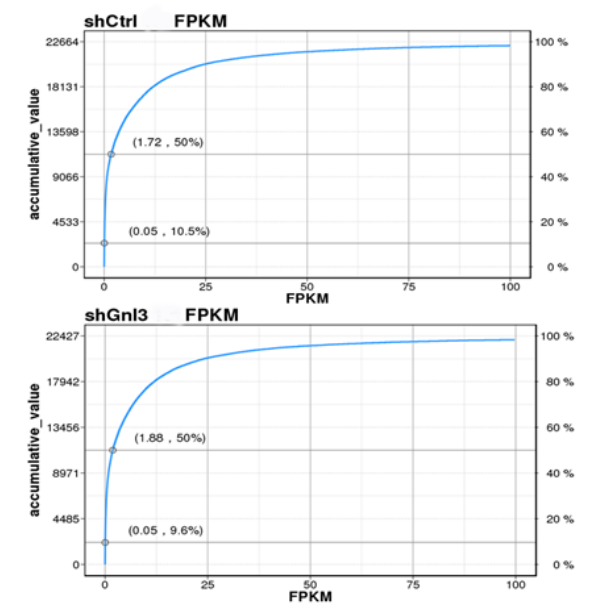

B

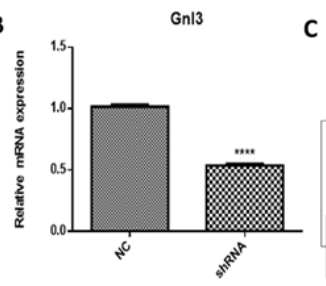

C
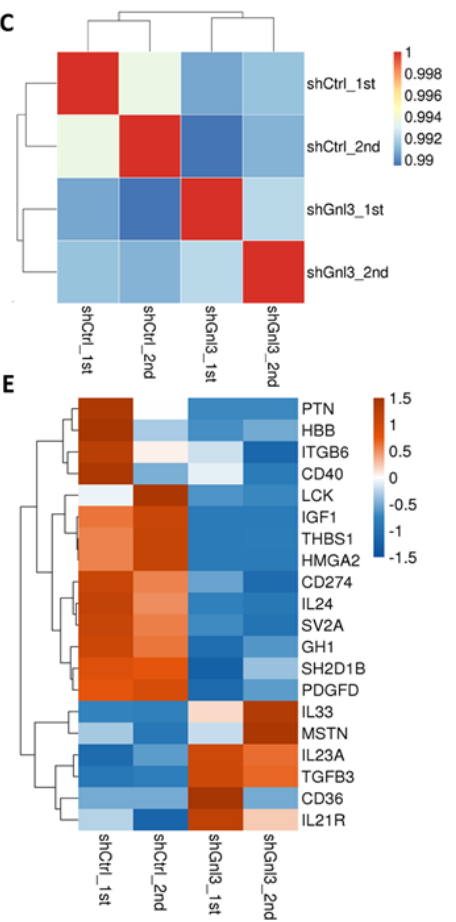

Figure 3. Functional clustering of GNL3-regulated genes. (A) The top ten representative GO biological processes of GNL3 down-regulated genes. (B) The top ten representative KEGG pathways of GNL3 downregulated genes. (C) IGV-sashimi plot shows a repression of IL24 expression after GNL3 knockdown. The exon sequences are denoted by boxes and intron sequences by the horizontal line. (D) IGV-sashimi plot shows a repression of PTN expression after GNL3 knockdown. (E) q-RT-PCR of IL24 gene in HeLa cells. (F) q-RT-PCR of PTN gene in HeLa cells. 


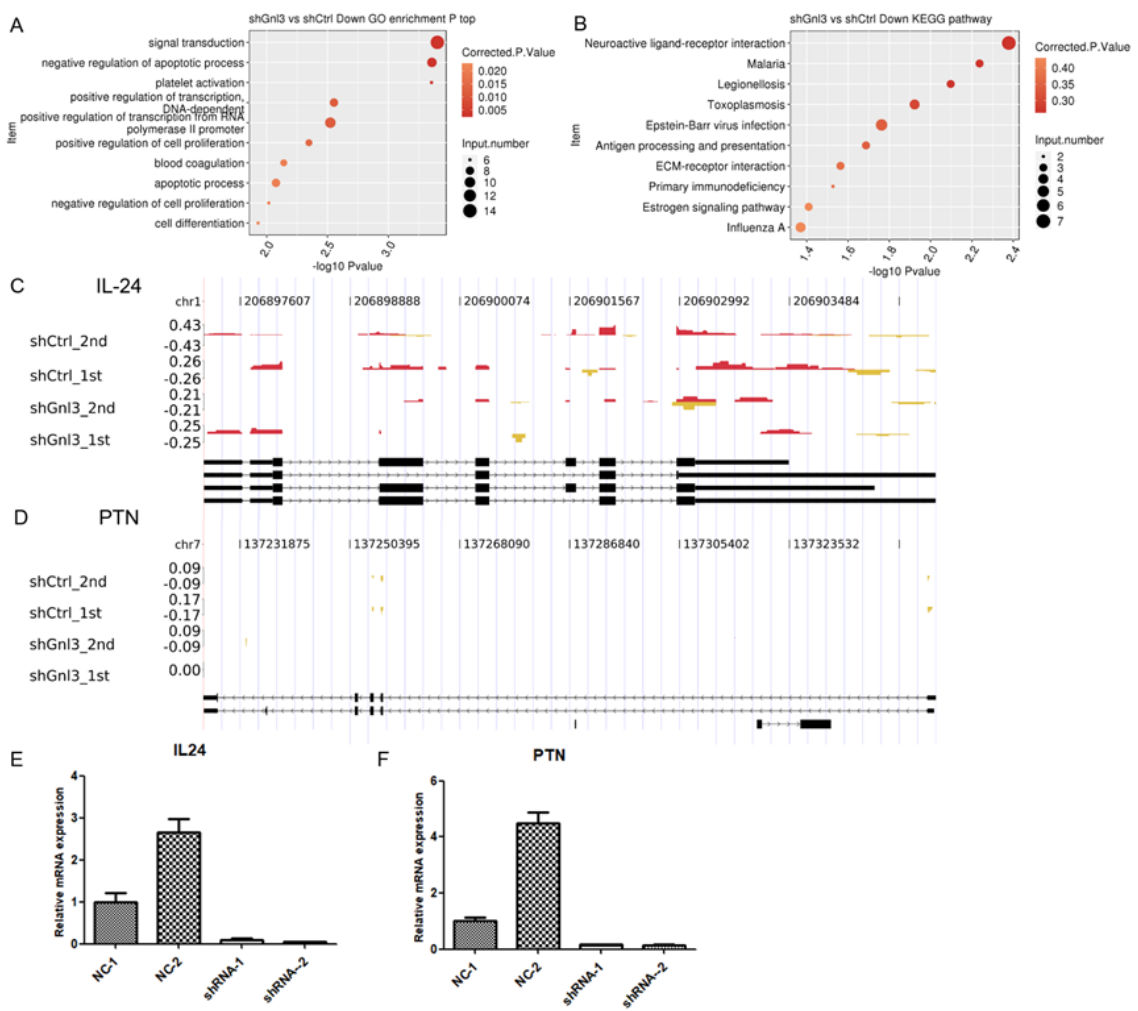

Figure 4. Expression level of GNL3, IL24 and PTN gene in the OA patients (A) The radiograph of the pelvis of a patient with OA shows narrowing of the hip joints. (B) The femoral head specimen removed from the patient with osteoarthritis shows degeneration of articular cartilage. (C) Western Blot in OA patients vs controls (D) q-RT-PCR of GNL3, IL24 and PTN gene in 20 patients with osteoarthritis and 20 control samples. P-value in GNL3, IL24 and PTN sets are respectively $0.0010,0.0230$ and $0.0073 .{ }^{*} \mathrm{P}<0.10,{ }^{* * *} \mathrm{P}$ $<0.01$ compared to the control groups. 


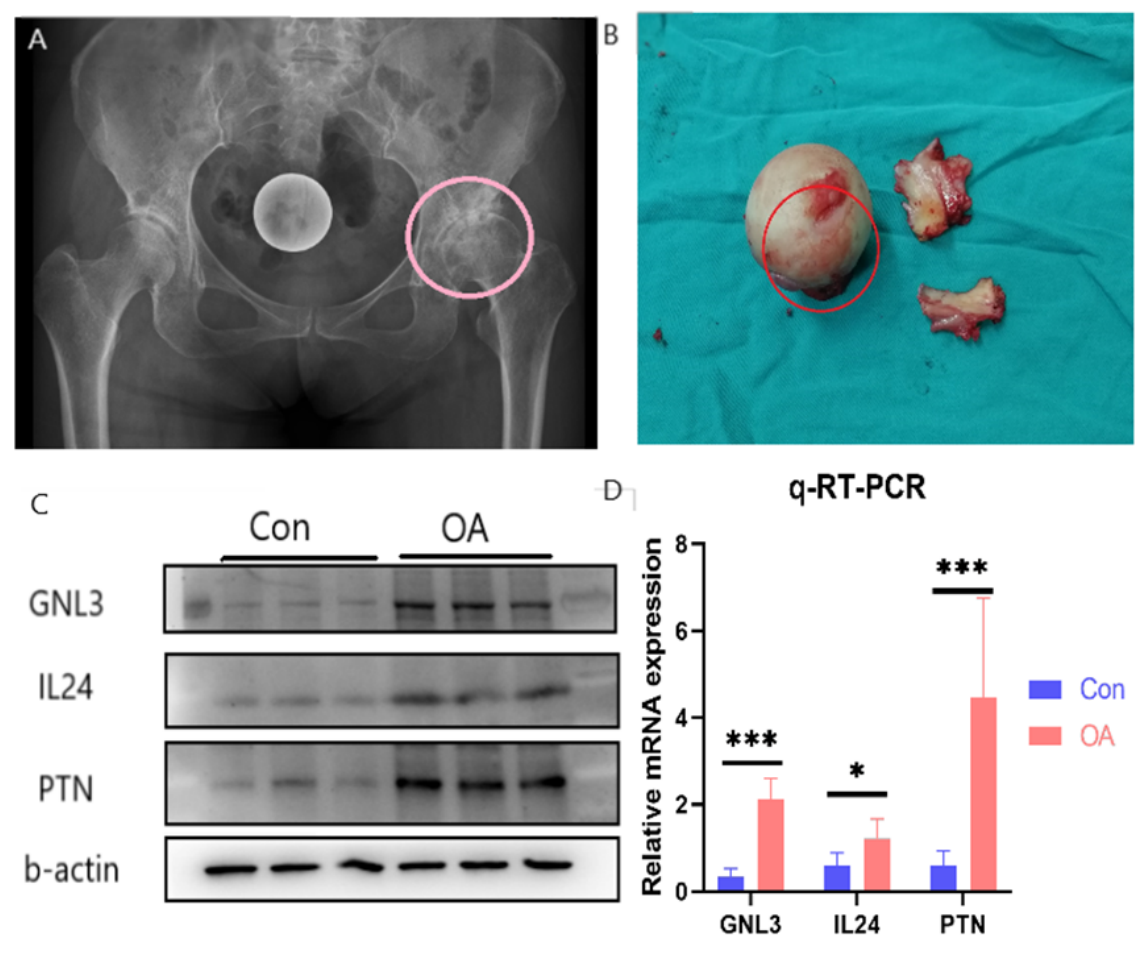

